\title{
Comparative Analysis of DWT and DWT-SVD Watermarking Techniques in RGB Images
}

\author{
Neha Narula ${ }^{1}$, Deepak Sethi ${ }^{2}$ and Partha Pratim Bhattacharya ${ }^{3}$ \\ 1, 2, 3 College of Engineering and Technology, Mody University of Science \& \\ Technology, Lakshmangarh, Rajasthan, India- 332311 \\ ${ }^{1}$ nehan.1991@gmail.com, ${ }^{2}$ deepaksethi@live.in, ${ }^{3}$ hereispartha@gmail.com
}

\begin{abstract}
Digital watermarking is an application associated with copyright protection. Any digital object can be used as a carrier to carry information. If the information is related to object then it is known as a watermark which can be visible or invisible. In the era of digital information, there are multiple danger zones like copyright and integrity violation of digital object. In case of any dispute during violation, content creator can prove ownership by recovering the watermark. In this paper, a comparative study of two most recent digital watermarking techniques namely DWT and DWT-SVD over RGB images is presented. In case of DWT (Discrete Wavelet Transform) watermarking technique, decomposition of the original image is done to embed the watermark and in case of DWTSVD watermarking technique, firstly original image is decomposed according to DWT and then watermark is embedded in singular values obtained by applying SVD (Singular Value Decomposition). The performances of the proposed techniques are compared on the basis of PSNR values.
\end{abstract}

Keywords: Digital Watermarking, Discrete Wavelet Transform (DWT), Singular Value Decomposition (SVD), Peak Signal to Noise Ratio (PSNR)

\section{Introduction}

Since the initial development of computer and network technology, the spread of digital multimedia information becomes easier in recent years. However, according to the insufficient awareness of the intellectual property, the condition of illegal copies and spread of copyright reserved information are growing serious. In order to raise the respect for the intellectual property, digital watermarking technique has been proposed as a method to embed an invisible or visible signal into multimedia data so as to check the owner identification of the data and discourage the unauthorized copying. Unlike encryption, which is useful for transmission but does not provide a way to examine the original data in its protected form, the watermark remains in the content in its original form and does not prevent a user from listening, viewing, examining, or manipulating the content. Also, unlike the idea of steganography, where the method of hiding the message may be secret and the message itself is secret, in watermarking, typically the watermark embedding process is known and the message does not has to be secret [1].

According to the domain in which the watermark is inserted, these techniques are classified into two categories, i.e., spatial domain and transform domain methods [2]. The spatial domain methods modify the digital data (pixels) directly to hide the watermark bits and possess the advantage of low computational complexity. On the other hand, the transform (frequency) domain methods do not alter the pixel values directly but rather modify the transform coefficients to hide the watermark bits such as Discrete Cosine Transform (DCT), Discrete Wavelet Transform (DWT) and Singular Value Decomposition (SVD) [3, 4]. 
The rest of the paper is organized as follows. The review of the related work is given in Section 2. The background review and the proposed algorithms for DWT and DWT-SVD are discussed in Section 3. The experimental results are given in Section 4. The performance analysis is given in Section 5. The conclusion and future work are given in Section 6.

\section{Related Works}

Review of literature survey has been conducted on discrete wavelet transformation and combination of discrete wavelet transformation with singular value decomposition techniques for hiding information in digital color images.

In [3], a hybrid image watermarking technique based on DWT and SVD has been presented where the watermark is embedded on the singular values of the cover image's DWT sub bands. The technique fully exploits the respective feature of these two transform domain methods: spatial-frequency localization of DWT and SVD efficiently represents intrinsic algebraic properties of an image. In [4], novel dual watermarking mechanism has been proposed for digital media that embeds a visible pattern into the spatial domain and an invisible logo into the frequency domain. The visible watermark indicates the ownership of the protected image through visual perception. Furthermore, even if the visible watermarked image incurs tampering, we can retrieve the invisible watermark to prove the copyright of the image. Only an authorized user can remove the dual watermarks to obtain an unmarked image with high fidelity. A hybrid image watermarking technique [5] has been presented for data hiding over Internet. The idea of the proposed technique is based on fusing multiple watermark images using wavelet fusion algorithm and embedding the resultant fused watermark in the original image using hybrid DWT-SVD watermarking algorithm to produce the watermarked image. The image watermarking technique using the hybrid DWT-SVD is more robust than that using the SVD only. The results also prove that the proposed watermarking technique improves both the capacity of the embedded information and robustness without affecting the perceptual quality of the original image. In [6], authors aimed at developing a hybrid image watermarking algorithm which satisfies both imperceptibility and robustness requirements. In order to achieve the objectives they have used singular values of wavelet transformation's HL and LH sub bands to embed watermark. A secret embedding key is designed to securely embed the fragile watermarks so that the new method is robust to counterfeiting, even when the malicious attackers are fully aware of the watermark embedding algorithm. The DWT and DWT-SVD watermarking schemes had been proposed [7] and there comparative study was done using the different PSNR values taken over different values of scale factor in gray scale images. In both DWT and DWTSVD, two-level decomposition was performed and then after decomposing the host and watermarking images, singular values of the images were modified using SVD over the sub-bands.

\section{Methodology}

\subsection{Discrete Wavelet Transform (DWT)}

Wavelet domain is a promising domain for watermark embedding. DWT is an orthogonal transform similar to the Discrete Cosine Transform that can be used for the audio and video compression, speech recognition, feature extraction, finger print, watermarking and many other applications in biomedical engineering [8]. This is a frequency domain technique in which firstly cover image is transformed into frequency domain and then its frequency coefficients are modified in accordance with the transformed coefficients of the watermark and watermarked image is obtained which is very much robust. In single level decomposition, DWT decomposes image hierarchically, 
providing both spatial and frequency description of the image. It decompose an image in basically three spatial directions i.e., horizontal, vertical and diagonal in result separating the image into four different components namely LL, LH, HL and HH. Here first letter refers to applying either low pass frequency operation or high pass frequency operations to the rows and the second letter refers to the filter applied to the columns of the cover image. LL level is the lowest resolution level which consists of the approximation part of the cover image. Rest three levels i.e., LH, HL, HH give the detailed information of the cover image [9].

3.1.1. Proposed DWT Watermarking Algorithm: The embedding algorithm for DWT based watermarking is shown in Figure 1. The algorithm works as follows:

Step 1: The original $\mathrm{N}^{*} \mathrm{~N}$ RGB image is transformed into sub-bands using single level 2-D DWT.

Step 2: The watermark of size $M * M$ RGB image is transformed into sub-bands using single level 2-D DWT.

Step 3: The resultant watermark is then embedded into the lower frequency sub-band of original image using the scale factor $(\alpha)$ i.e., $W I=O+\alpha W$

Step 4: Finally, inverse 2-D DWT is performed to produce the watermarked image.

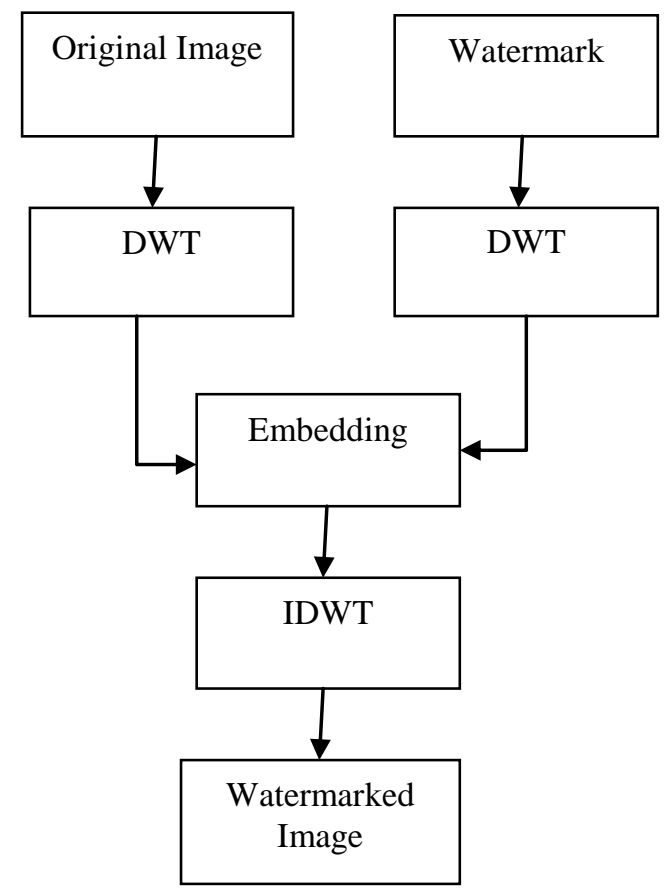

Figure 1. DWT based Embedding

The extraction algorithm for DWT based watermarking is shown in Figure 2. The algorithm works as follows:

Step 1: The original $\mathrm{N}^{*} \mathrm{~N}$ RGB image is transformed into sub-bands using single level 2-D DWT.

Step 2: The watermark of size $M^{*} M$ RGB image is transformed into sub-bands using single level 2-D DWT.

Step 3: The watermarked image (output of embedding) is transformed into sub-bands using the single level 2-D DWT.

Step 4: Then the extraction is applied to the decomposed watermarked image using the same value of scale factor $(\alpha)$ i.e., $E W I=(W M-O) / \alpha$

Step 5: Finally, inverse 2-D DWT is performed to get the extracted watermark image. 


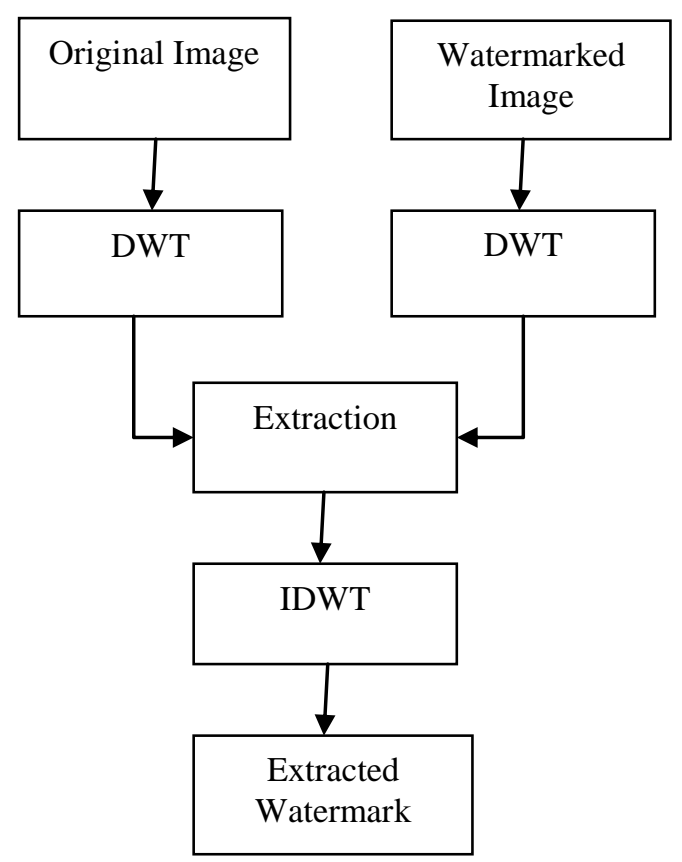

Figure 2. DWT based Extraction

\subsection{Singular Value Decomposition (SVD)}

Singular Value Decomposition is a linear algebra transform which is used for factorization of a real or complex matrix with numerous applications in various fields of image processing. As a digital image can be represented in a matrix form with its entries giving the intensity value of each pixel in the image, SVD of an image $M$ with dimensions $\mathrm{m} x \mathrm{~m}$ is given by [10]:

$$
M=U S V^{T}
$$

Where, $\mathrm{U}$ and $\mathrm{V}$ are orthogonal matrices and $\mathrm{S}$ known as singular matrix is a diagonal matrix carrying non-negative singular values of matrix $\mathrm{M}$. The columns of $\mathrm{U}$ and $\mathrm{V}$ are called left and right singular vectors of $\mathrm{M}$, respectively. They basically specify the geometry details of the original image. Left singular matrix i.e., $\mathrm{U}$ represents the horizontal details and right singular matrix i.e., $\mathrm{V}$ represents the vertical details of the original image. The diagonal values of matrix $\mathrm{S}$ are arranged in decreasing order which signifies that the importance of the entries is decreasing from first singular value to the last one. This feature is employed in SVD based compression techniques.

There are two main properties of SVD to employ in digital watermarking schemes [11]:

1. Small variations in singular values do not affect the quality of image.

2. Singular values of an image have high stability.

\subsection{Hybrid DWT-SVD}

Hybrid technique is a fusion of two techniques. Here, DWT and SVD are used together to improve the quality of digital watermarking and hence increases the robustness and imperceptibility of an image.

3.3.1. Proposed Hybrid DWT-SVD Watermarking Algorithm: The embedding algorithm for DWT-SVD based watermarking is shown in Figure 3. The algorithm works as follows: 


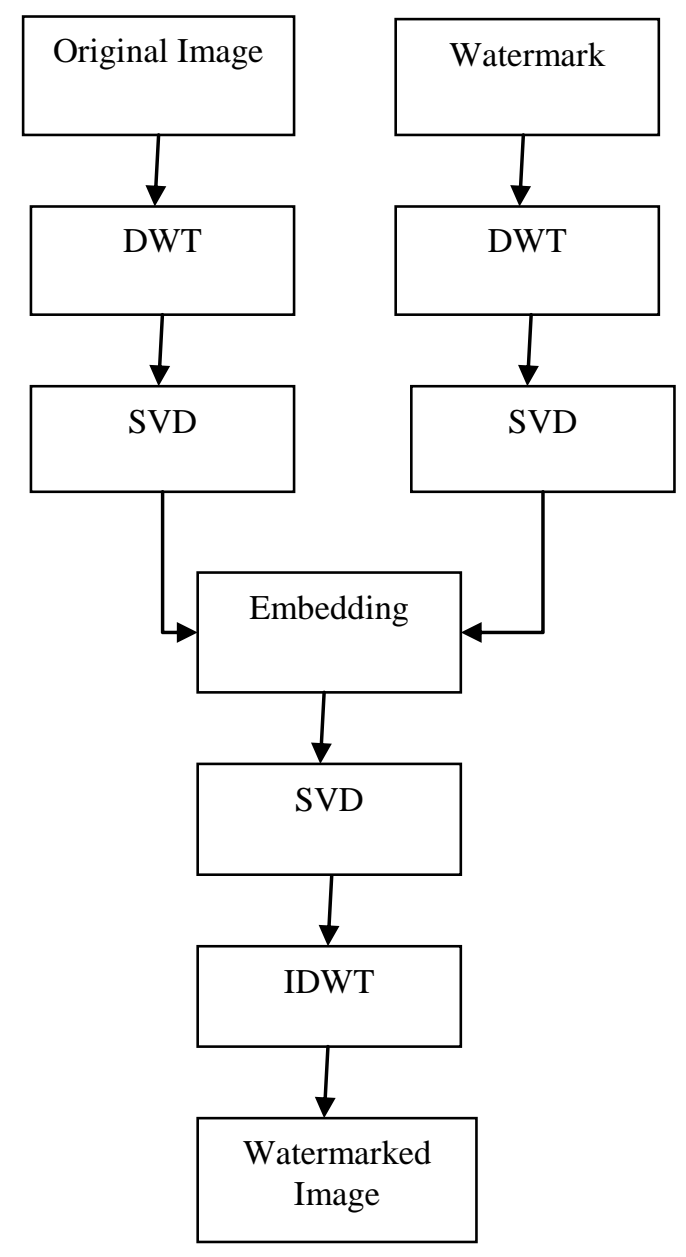

Figure 3. DWT-SVD based Embedding

Step 1: The original $\mathrm{N}^{*} \mathrm{~N}$ RGB image is transformed into sub-bands using single level 2-D DWT.

Step 2: SVD is performed on LL sub-band (on RGB components) of decomposed RGB original image i.e., $S=U S V^{T}$

Step 3: The watermark of size $M * M$ RGB image is transformed into sub-bands using single level 2-D DWT.

Step 4: SVD is performed on LL sub-band (on RGB components) of decomposed RGB watermark image i.e., $S W=U_{w} S_{w} V_{w}^{T}$

Step 5: After performing SVD on both original and watermark images, the resultant watermark image is then embedded with the original image using the scale factor $(\alpha)$ i.e.,

$S W I=S+\alpha(S W)$

Step 6: Inverse SVD is performed on embedded image.

Step 7: Finally, inverse 2-D DWT is performed to produce the watermarked image.

The extraction algorithm for DWT-SVD based watermarking is shown in Figure 4. The algorithm works as follows: 


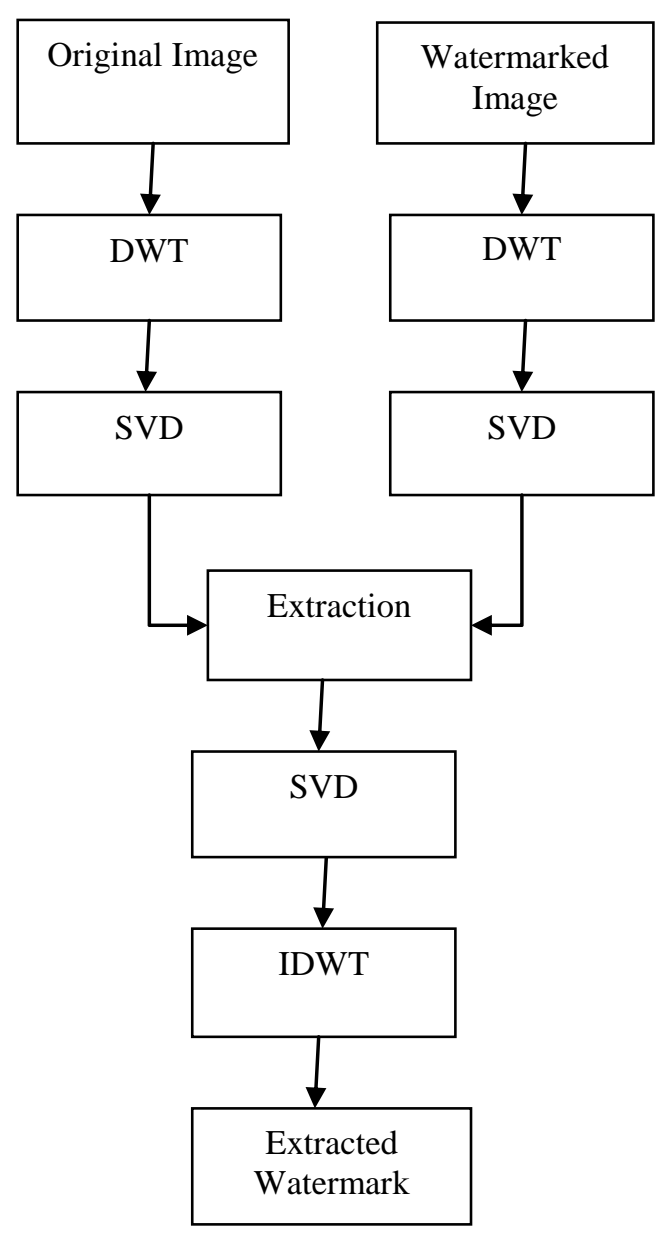

Figure 4. DWT-SVD based Extraction

Step 1: The original $\mathrm{N}^{*} \mathrm{~N}$ RGB image is transformed into sub-bands using single level 2-D DWT.

Step 2: SVD is performed on LL sub-band (on RGB components) of decomposed RGB original image i.e., $\mathrm{S}=\mathrm{USV}^{\mathrm{T}}$

Step 3: The watermark of size $\mathrm{M}^{*} \mathrm{M}$ RGB image is transformed into sub-bands using single level 2-D DWT.

Step 4: SVD is performed on LL sub-band (on RGB components) of decomposed RGB watermark image i.e., $S W=U_{w} S_{w} V_{w}^{T}$

Step 5: The watermarked image (output of embedding) is transformed into sub-bands using the single level 2-D DWT.

Step 6: SVD is performed on LL sub-band (on RGB components) of decomposed RGB watermarked image i.e., $S W I=U_{w} S_{w} V_{w}^{T}$

Step 7: Then the extraction is applied to the resultant SVD image using the same value of scale factor $(\alpha)$ i.e., $E W I=(S W I-S) / \alpha$

Step 8: Inverse SVD is applied on resultant image after extraction.

Step 9: Finally, inverse 2-D DWT is performed to get the extracted watermark image.

In this Section, we have designed and explained the Steps included in DWT and DWTSVD watermarking techniques. The results of these techniques will be shown in Section 4. 


\section{Experimental Results}

In this Section, we have demonstrated the practicability of the proposed watermarking approaches. Moreover, we compare the DWT with DWT-SVD in terms of image imperceptibility and robustness. Test images are RGB images with size $512 \times 512$ pixels. The proposed DWT and hybrid DWT-SVD image watermarking techniques are coded using MATLAB. Figure 5 shows the cover image (RGB image) and Figure 6 shows the watermark image (RGB image). Initially, both cover and watermark images are used in embedding and extraction algorithms. Figure 7 shows the watermarked image based on DWT and Figure 8 shows the watermarked image based on DWT-SVD as a result of embedding algorithms in both the techniques. Figure 9 shows the extracted watermark image based on DWT and Figure 10 shows the extracted watermark image based on DWT-SVD as a result of extraction algorithms in both techniques.

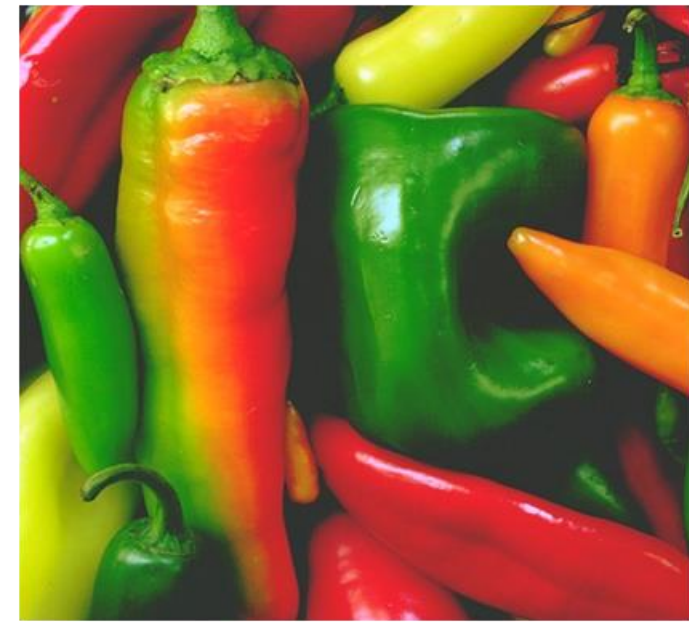

Figure 5. Cover Image

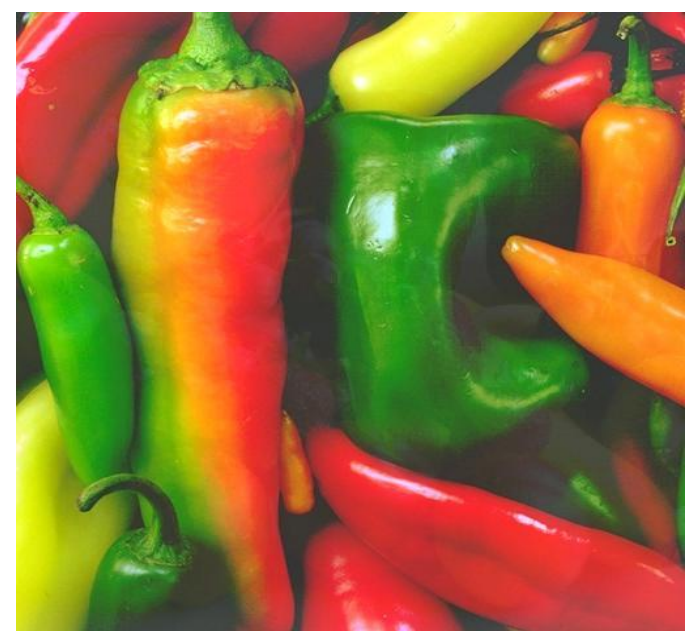

Figure 7. Watermarked Image based on DWT

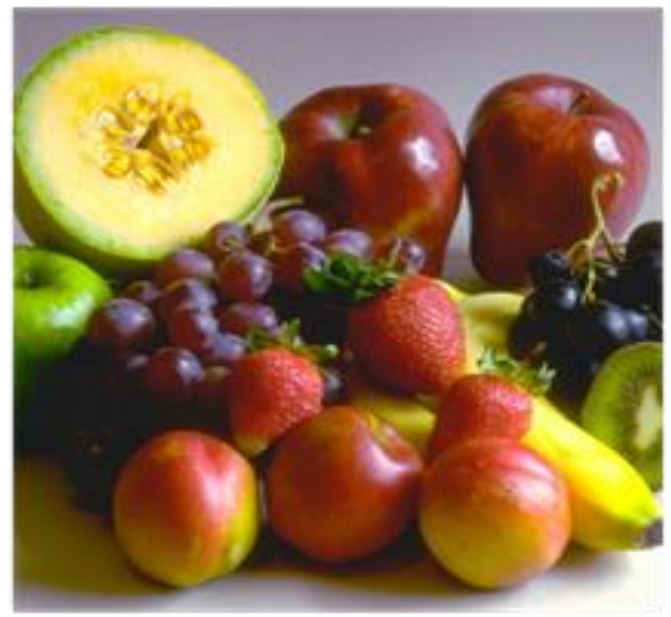

Figure 6. Watermark Image

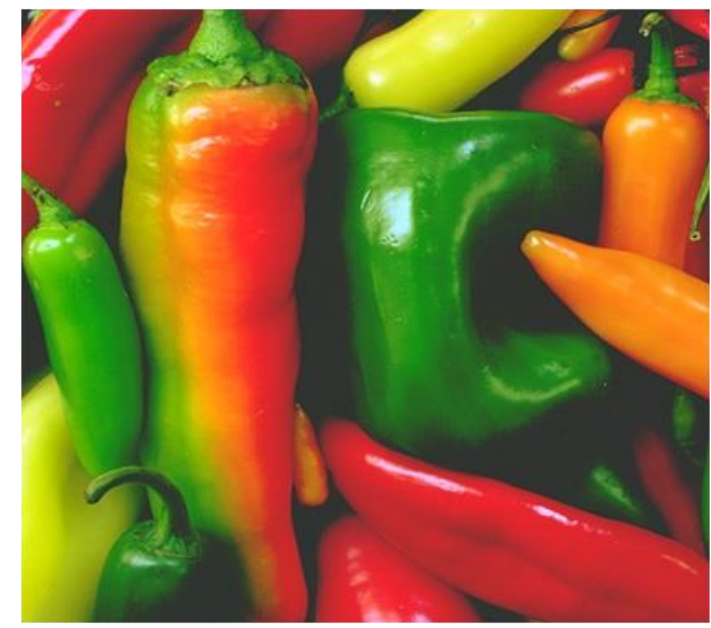

Figure 8. Watermarked Image based on DWT-SVD 


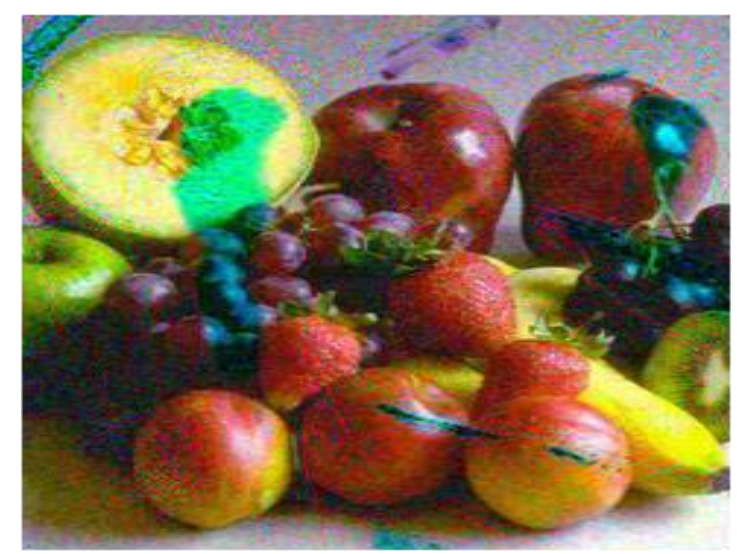

Figure 9. Extracted Watermark Image based on DWT

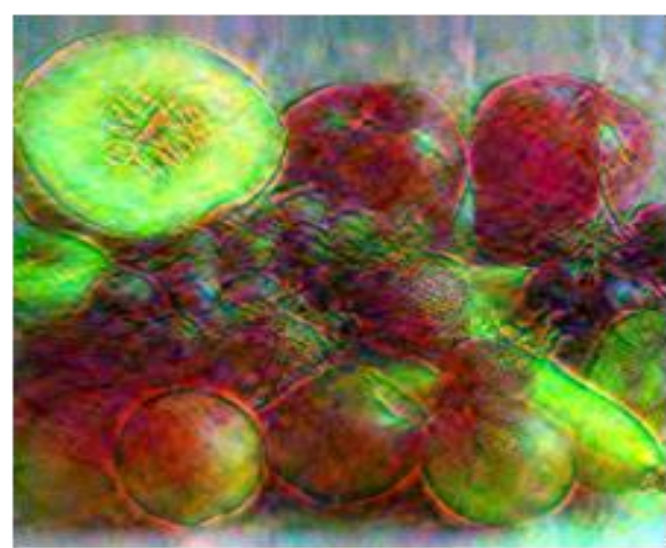

Figure 10. Extracted Watermark Image based on DWT-SVD

\section{Performance Analysis}

In all experiments, the quality of the watermarked image is evaluated by applying Peak Signal to Noise Ratio (PSNR) as image quality metrics. The quality of watermarked image is measured in terms of PSNR (Peak Signal to Noise Ratio) and MSE (Mean Square Error). In ideal case, PSNR should be infinite and MSE should be zero. But this is not possible for watermarked image, so large PSNR and small MSE is desirable.

$$
\begin{aligned}
& M S E=1 / M N \sum_{i=1}^{m} \sum_{j=1}^{n}\left[I(i, j)-I^{\prime}(i, j)\right]^{2} \\
& P S N R=10 \log 10[(\max ) \quad 2 / M S E]
\end{aligned}
$$

where $\mathrm{N}^{*} N$ is the dimension of the images; $I(i, j)$ is the original image; $I^{\prime}(i, j)$ is the watermarked image and max is the maximum intensity of the original image.

Table I shows the comparison between PSNR values of DWT and DWT-SVD. Figure 10 shows the graph plot for comparison for PSNR values (from eqn. 5.2) with respect to scaling factor $(\alpha)$ between DWT and DWT-SVD.

Table I. Comparison between PSNR Values of DWT and DWT-SVD

\begin{tabular}{|c|c|c|}
\hline $\begin{array}{c}\text { Value of Scaling } \\
\text { factor( } \boldsymbol{\alpha})\end{array}$ & \multicolumn{2}{|c|}{ Value of PSNR } \\
\cline { 2 - 3 } & DWT & DWT-SVD \\
\hline 0.01 & 35.82 & 35.87 \\
\hline 0.02 & 34.78 & 35 \\
\hline 0.03 & 33.45 & 33.74 \\
\hline 0.04 & 32.13 & 32.44 \\
\hline 0.05 & 30.82 & 31.20 \\
\hline 0.06 & 29.67 & 30.11 \\
\hline 0.07 & 28.58 & 29.09 \\
\hline 0.08 & 27.62 & 28.15 \\
\hline 0.09 & 26.70 & 27.31 \\
\hline 0.10 & 25.90 & 26.54 \\
\hline
\end{tabular}




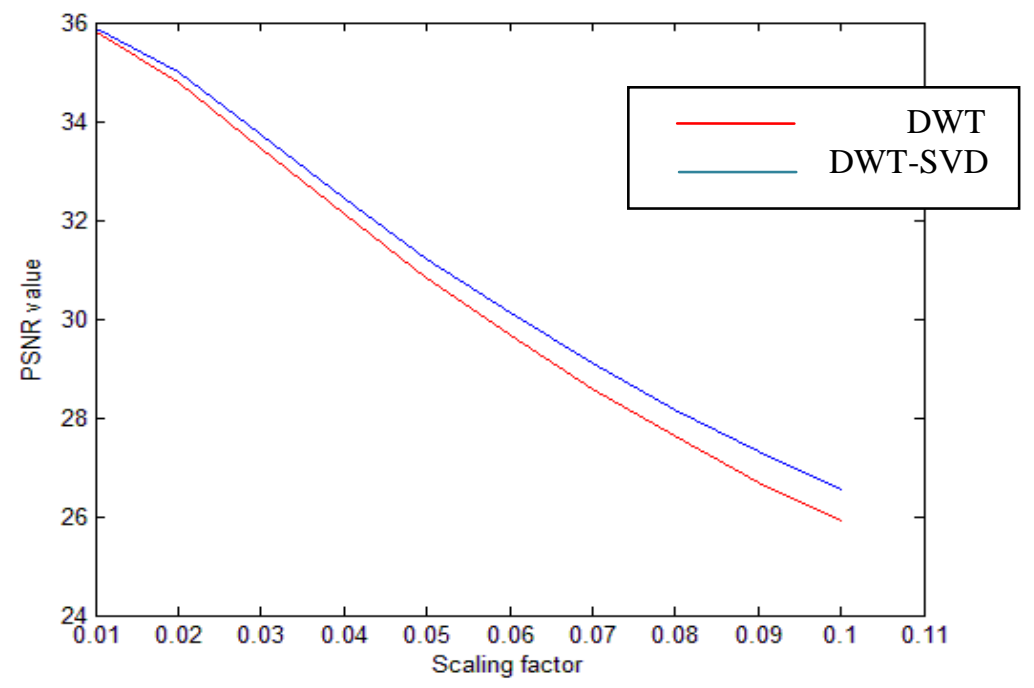

\section{Figure 11. Graph Plot for Comparison between PSNR Values of DWT and DWT-SVD}

\section{Conclusion and Future Work}

The proposed DWT and DWT-SVD watermarking technique are applied to ensure the copyright protection and security of content or images and to increase the robustness of image. After applying both the watermarking techniques and comparing the values of PSNR at different values of scaling factor $(\alpha)$, it is concluded that the hybrid DWT-SVD technique is much better than DWT technique as at every value of scaling factor, value of peak signal to noise ratio (PSNR) is more in case of the hybrid DWT-SVD technique. Less the value of PSNR more will be the degradation in the quality of the original image. This shows that after watermarking, the quality of original image degrades more when DWT technique is used for embedding the watermark in comparison with DWT-SVD technique embedding.

Since the size of the color image is reducing more as a result of applying DWT-SVD watermarking approach, therefore this technique may be used in various applications of wireless sensor networks as a future scope.

\section{Acknowledgment}

Authors would like to thank Mody University of Science \& Technology, Lakshmangarh. The authors also like to thank the anonymous reviewers for the helpful comments and suggestions.

\section{References}

[1] C. I. Podilchuk and E. J. Delp, "Digital Watermarking: Algorithms and Applications", IEEE Signal Processing Magazine, (2001) July.

[2] V. Santhi and A. Thangavelu, "DWT-SVD Combined Full Band Robust Watermarking Technique for Color Images in YUV Color Space", International Journal of Computer Theory and Engineering, vol. 1, no. 4, (2009) October.

[3] C.-C. Lai, Member and C.-C. Tsai, "Digital Image Watermarking Using Discrete Wavelet Transform and Singular Value Decomposition”, IEEE Transactions on Instrumentation and Measurement, vol. 59, no. 11, (2010) November.

[4] P.-Y. Lin, J.-S. Lee and C.-C. Chang, "Dual Digital Watermarking for Internet Media Based on Hybrid Strategies", IEEE Transactions on Circuits and System for Video Technology, vol. 19, no. 8, (2009) August.

[5] E. E.-D. Hemdan, N. El-Fishaw, G. Attiya and F. A. El-Samii, "Hybrid Digital Image Watermarking Technique for Data Hiding”, IEEE 30th National Radio Science Conference, (2013). 
[6] S. Ramakrishnan, T. Gopalakrishnan and K. Balasamy, "SVD Based Robust Digital Watermarking For Still Images Using Wavelet Transform”, CCSEA 2011, CS \& IT, (2011), pp. 155-167.

[7] N. Bisla and P. Chaudhary, "Comparative Study of DWT and DWT-SVD Image Watermarking Techniques", IJARCSSE, vol. 3, (2013) June, pp. 821-825.

[8] M. V. Malakooti, Z. F. Panah and S. M. Hashemi, "Image Recognition Method based on Discrete Wavelet Transform (DWT) and Singular Value Decomposition (SVD)", SDIWC, (2013).

[9] B. Ram, "Digital Image Watermarking Technique Using Discrete Wavelet Transform and Discrete Cosine Transform", International Journal of Advancements in Research \& Technology, vol. 2, Issue 4, (2013) April.

[10] P. Singh and S. Agarwal, "A Hybrid DCT-SVD Based Robust Watermarking Scheme for Copyright Protection", International Conference on Emerging Trends in Engineering and Technology (ICETET), (2013).

[11] V. Santhi and A. Thangavelu, "DWT-SVD Combined Full Band Robust Watermarking Technique for Color Images in YUV Color Space”, International Journal of Computer Theory and Engineering, vol. 1, no. 4, (2009) October.

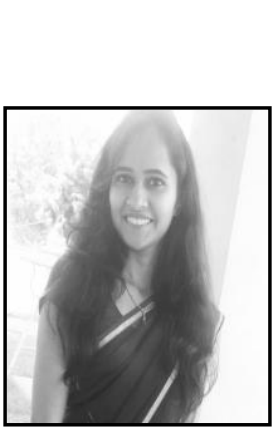

\section{Authors}

Neha Narula was born in India on August 19, 1991. She received her B. Tech. degree from Mody University of Science and Technology and presently pursuing her M. Tech. from Mody University, Lakshmangarh. Her current research interests include digital watermarking, image protection and information security in wireless sensor networks.

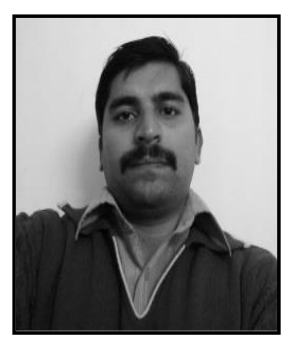

Deepak Sethi was born in India on November 11, 1985. He is working as an Assistant Professor at Mody University, Laxmangarh, and Rajasthan, INDIA in the department of Computer Science and Engineering. He has completed his M. Tech. in Computer Science and Engineering from DCRUST Murthal, INDIA in 2011. He is currently doing research on secure and efficient routing in wireless sensor network under the supervision of Prof. P.P. Bhattacharya. Also, image processing and software engineering are the key fields of his research.

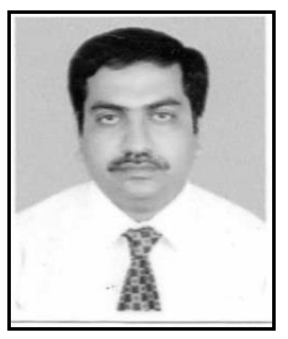

Partha Pratim Bhattacharya was born in India on January 3, 1971. He has 16 years of experience in teaching and research. He served many reputed educational Institutes in India in various positions. At present he is working as Professor in Department of Electronics and Communication Engineering in the College of Engineering and Technology, Mody University, Rajasthan, India. He worked on Microwave devices and systems and mobile cellular communication systems. He has published more than 90 papers in refereed journals and conferences. His present research interest includes mobile cellular communication, wireless sensor network and cognitive radio. Dr. Bhattacharya is a member of The Institution of Electronics and Telecommunication Engineers, India and The Institution of Engineers, India. He is the recipient of Young Scientist Award from International Union of Radio Science in 2005. He is working as the chief editor, editorial board member and reviewer in many reputed journals. 